\title{
Future plans at COMPASS
}

\author{
J. Pretz ${ }^{\mathrm{a}}$ on behalf of the COMPASS collaboration \\ Physikalisches Institut, Universität Bonn, Nußallee 12, 53115 Bonn, Germany
}

\begin{abstract}
After successfully running since several years with polarized muon and hadron beams to study the spin structure of the nucleon and spectroscopy of hadrons, the COMPASS (Common Muon and Proton Apparatus for Structure and Spectroscopy) experiment enters a second phase with a new extended physics program to obtain deeper insight in the partonic structure of matter. These future plans include a measurement of deep virtual Compton scattering in order to determine generalized parton distributions and the study of the polarized DrellYan process giving access to transverse momentum dependent (TMD) parton distributions. Recent results and these future plans will be presented in this document.
\end{abstract}

\section{Motivation}

\subsection{Partonic structure of the nucleon}

Most of our knowledge about the internal structure of the nucleon comes from lepton-nucleon scattering experiment. Starting with Hofstadter's experiment[1] in 1955 proving that the proton has a finite radius, culminating in the manifestation of pointlike particles identified as quarks by Friedman, Kendall and Taylor in deep inelastic scattering [2].

At high momentum transfers from the lepton to the nucleon the nucleon can be described in terms of parton distribution functions. At leading twist and after integration over the transverse momentum of the quarks inside the nucleon the nucleon is fully described by three parton distributions, the unpolarized parton distributions $q(x)$ and $g(x)$, the helicity distributions $\Delta q(x)$ and $\Delta g(x)$ and the transversity distribution $\Delta_{T} q(x)$. Note that there is no corresponding gluon transversity distribution $\Delta_{T} g(x)$ in a spin $1 / 2$ particle. Figure 1 shows these distributions in a pictorial way. They have simple probabilistic interpretations. The unpolarized distributions $q(x) \mathrm{dx}$ equals the number of quarks of type $q$ carrying a longitudinal momentum fraction in the interval $[x, x+\mathrm{dx}]$ inside the nucleon. The helicity distribution gives the difference of the number of quarks having their spin aligned with the nucleon spin and the number of quarks with spin anti-aligned in a longitudinally polarized nucleon. A similar interpretation holds for the transversity distribution in a transversally polarized nucleon.

Helicity distributions can be measured by analyzing double spin asymmetries of longitudinally polarized lepton beams and longitudinally polarized targets. The transversity distribution is related to a single spin Collinsasymmetry on a transversally polarized target.

The simple partonic interpretation can be extended if one does not integrate over the transverse momentum of

\footnotetext{
a e-mail: jorg.pretz@cern.ch
}

$$
\begin{aligned}
& q(x), g(x) \\
& q=u, d, s, \bar{u}, \bar{d}, \bar{s} \\
& \Delta q(x), \Delta g(x) \text { (helicity) } \\
& \Delta_{T} q(x) \text { (transversity) }
\end{aligned}
$$

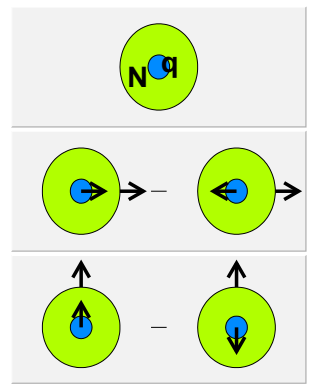

Fig. 1. The three twist three parton distributions appearing in the cross section for lepton-nucleon scattering after integration over

\begin{tabular}{|c|c|c|c|}
\hline quark $\quad$ nucleon & unpol. & long. & trans. \\
\hline unpol. & $f_{1}(q)$ & & $f_{1 T}^{\perp}\left(q_{T}\right)$ \\
\hline long. & & $g_{1}(\Delta q)$ & $g_{1 T}$ \\
\hline trans. & $h_{1}^{\perp}$ & $h_{1 L}$ & $h_{1}\left(\Delta_{T} q\right), h_{1 T}^{\perp}$ \\
\hline
\end{tabular}
transverse momentum.

Table 1. All eight leading twist parton distributions. Distributions in parentheses denote alternative notations found in the literature.

the quarks. In this case five more parton distributions appear in the cross section at leading twist. These are referred to as transverse momentum dependent (TMD) distributions in the literature. Table 1 gives an overview [15]. The TMD distributions can be accessed in deep inelastic scattering studying the azimuthal distribution of the produced hadrons or in Drell-Yan processes.

Another extension of the simple collinear quark parton model is to consider the transverse position of a quark inside the nucleon. This effect is described by generalized parton distributions accessible in deep virtual Compton scattering. These distributions describe the probability that a parton carries a certain longitudinal momentum fraction at 
a given transverse position in the nucleon as indicated by Fig. 2.

Investigation of generalized parton distributions (GPDs) and transverse momentum distributions (TMDs) is part of the future COMPASS programme.

\subsection{Theoretical Background}

All the distributions discussed are related to the following matrix element of nucleon states:

$$
<P^{\prime}|\bar{\Psi}(\eta) \hat{O} \Psi(0)| P>
$$

The following cases are of interest

- $P=P^{\prime}$ are related to the quark distributions. Depending on the operator $\hat{O}$ chosen, one has access to the various distributions in Tab. 1

$-\eta=0$ is related to form factors

- the most general case $P \neq P^{\prime}$ and $\eta \neq 0$ are related to generalized parton distributions

Here are two explicit examples [3]:

$$
\begin{gathered}
\frac{1}{2} \int \frac{\mathrm{d} \eta_{\perp} \mathrm{d} \eta^{-}}{(2 \pi)^{3}} \mathrm{e}^{\mathrm{k}^{+} \cdot \xi^{-}+\mathrm{k}_{\perp} \cdot \eta_{\perp}}<\mathrm{P}\left|\bar{\Psi}(0) \gamma^{+} \Psi\left(\eta^{-}, \eta_{\perp}\right)\right| \mathrm{P}> \\
=q\left(x, k_{\perp}\right)+q_{T}\left(x, k_{\perp}\right)\left(\hat{k} \times \hat{S}_{T}\right) \cdot \hat{P}
\end{gathered}
$$

This matrix element contains information on the ordinary unpolarized parton distribution $q\left(x, k_{\perp}\right)$ and the Sivers function $q_{T}\left(x, k_{\perp}\right) . S_{T}$ denotes the transverse polarization of the nucleon.

The matrix element

$$
\begin{aligned}
& \int \frac{\mathrm{dx}^{-}}{4 \pi} \mathrm{e}^{\mathrm{ip}^{+} \mathrm{x}^{-}}\left\langle\mathrm{P}^{\prime}\left|\bar{\Psi}\left(-\eta^{-} / 2\right) \gamma^{+} \Psi\left(\eta^{-} / 2\right)\right| \mathrm{P}\right\rangle \\
& =H_{q}(x, \xi, t) \frac{1}{2 p^{+}} \bar{u}\left(P^{\prime}\right) \gamma^{+} u(P) \\
& \quad+E_{q}(x, \xi, t) \frac{1}{2 p^{+}} \bar{u}\left(P^{\prime}\right) \frac{i \sigma^{+v}\left(P-P^{\prime}\right)_{v}}{2 M} u(P)
\end{aligned}
$$

is parameterized by two GPDs, $H_{q}$ and $E_{q} \cdot \xi \approx x /(2-x)$ is the skewness and $t=\left(P-P^{\prime}\right)^{2}$ the momentum transfer to the nucleon.

From the definitions above one can read off the relation between GPDs and from factors $\left(F_{1}(t)\right)$ and parton distributions:

$$
\begin{aligned}
H(x, 0,0) & =q(x) \\
\int H(x, \xi, t) \mathrm{dx} & =F_{1}(t) \quad \forall \xi
\end{aligned}
$$

\section{Results from the COMPASS Experiment}

COMPASS is a fixed target experiment at CERN using hadron and muon beams to study the structure of the nucleon and spectroscopy of hadrons. In this section the COMPASS experiment will be described and recent selected results on longitudinal and transverse spin structure will be presented.

\subsection{The COMPASS Experiment}

The main experimental requirements to perform the measurements discussed above are

- a high energetic polarized lepton beam

- a polarized target

- good particle identification

The COMPASS experiments meets these requirements. A $160 \mathrm{GeV}$ naturally polarized positively charged muon beam is used. Its polarization is $-0.80 \pm 0.04$. ${ }^{6} \mathrm{LiD}$ (containing polarizable deuterons) or $\mathrm{NH}_{3}$ (polarized protons) serve as target material. The target material is placed in a superconducting solenoid and is polarized via dynamic nuclear polarization. ${ }^{6} \mathrm{Li}$ can be considered as a helium nucleus plus a deuteron. Thus 4 out of the 8 nucleons in ${ }^{6} \mathrm{LiD}$ are polarizable. This would lead to a so called dilution factor of $4 / 8=0.5$. The corresponding dilution factor for $\mathrm{NH}_{3}$ is $3 / 17 \approx 0.18$. Talking into account additional non polarizable material in the target leads to a dilution factor of approximately $0.4(0.15)$ for the ${ }^{6} \mathrm{LiD}\left(\mathrm{NH}_{3}\right)$ target. The exact value depends on the kinematics and is calculated event by event. Its relative uncertainty is $5 \%$. The target polarization reached is $50 \%(85 \%)$ with a relative error of $5 \%$ for the deuteron (proton) target. For the years 2002-2004 two target cells oppositely polarized were in use. In 2006 and 2007, 3 target cells were employed, the outer two had opposite polarization compared to the central cell. The proton target was only in in operation in the year 2007. The use of 2 (or 3 ) target cells oppositely polarized and simultaneously exposed to the muon beam is mandatory for the extraction of asymmetries. Combining data sets with two different polarizations with respect to the muon polarization, allows, to a large extend, a cancellation of possible acceptance variations during the data taking.

The scattered muon and the produced hadrons are detected and identified in a two stage magnetic spectrometer. Electromagnetic and hadron calorimeters and a Ring Imaging Cherenkov (RICH) Detector are used for particle identification. The RICH allows a pion-kaon separation from threshold $(9 \mathrm{GeV})$ up to momenta of $40 \mathrm{GeV}$ at a $2.5 \sigma$ level. For a more detailed description of the experimental setup see [4]. A schematic view of the spectrometer is shown in Fig. 3.

\subsection{Recent Results}

During the periods of data taking with a muon beam, approximately $80 \%$ of the time was dedicated to run with a longitudinally polarized target, the remaining $20 \%$ with transversally polarized target.

The main goal of the measurement with a longitudinally polarized target is the determination of the gluon polarization $\Delta g / g$. The gluon polarization is accessible via double spin asymmetries selecting specific hadronic final states which enhance the number of partonic processes where a gluon participated (s. Fig. 4).

These are hadron pairs with large transverse momentum or charmed mesons. Figure 5 shows a summary of 


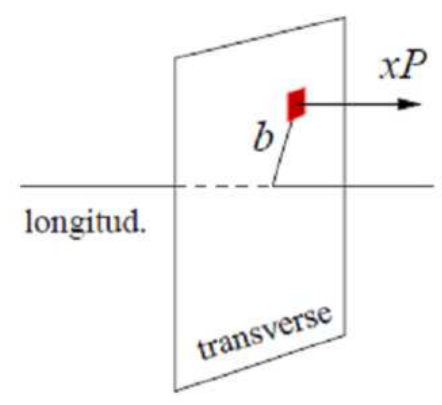

(a)

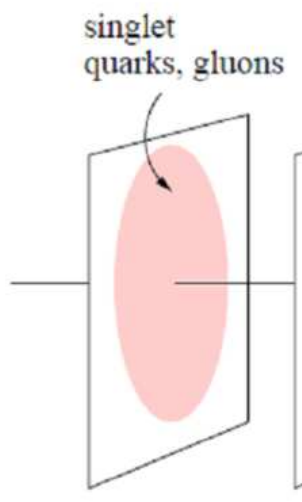

(b) $x<0.01$

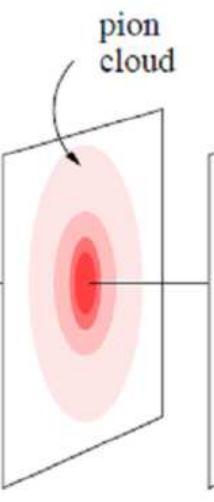

$x \sim 0.1$

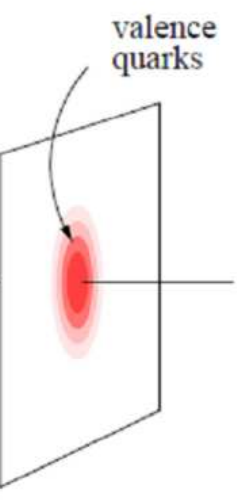

$x \sim 0.3$

Fig. 2. Interpretation of generalized parton distributions as correlated longitudinal momentum and transverse space distributions.

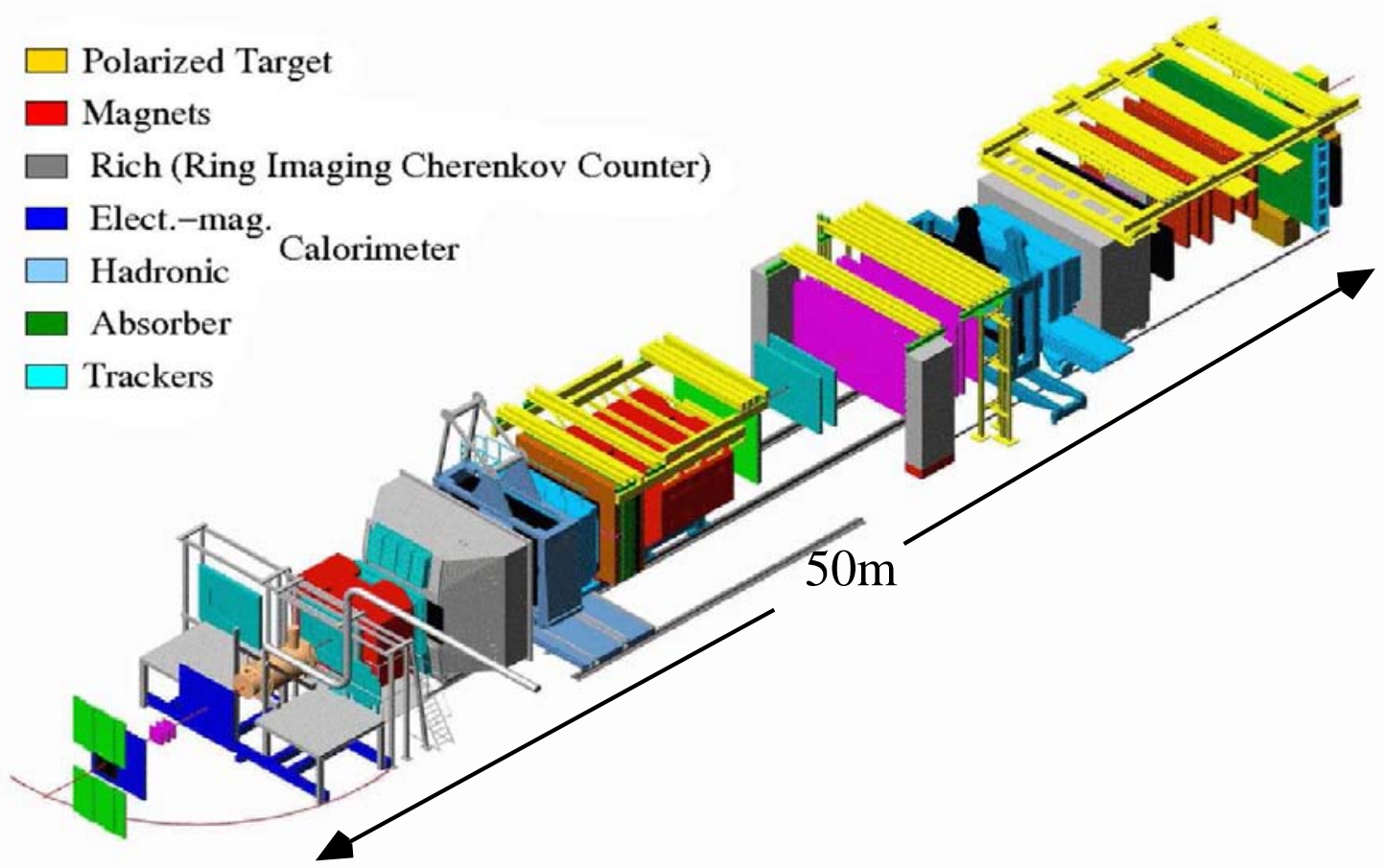

Fig. 3. The Compass spectrometer.

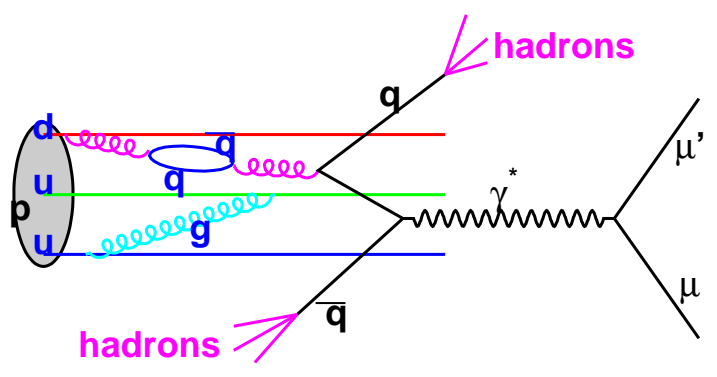

Fig. 4. Deep Inelastic events with the photon-gluon fusion process as underlying partonic subprocess. recent measurements of $\Delta g / g$ from COMPASS and other experiments. All the results are consistent with a vanishing gluon polarization at a gluon momentum fraction of $x_{g} \approx 0.1$, although the first moment $\Delta G=\int_{0}^{1} \mathrm{dx}_{\mathrm{g}} \Delta \mathrm{g}\left(\mathrm{x}_{\mathrm{g}}\right)$ is still consistent with a value of 0.5 which would mean that the gluon helicity accounts for $100 \%$ of the nucleon spin.

Figure 6 shows results from measurements of the quark helicity distributions [9]. These measurements are performed in parallel to the measurement of $\Delta \mathrm{g} / \mathrm{g}$. Here double spin asymmetries of various hadron species $\left(\pi^{+}, \pi^{-}, K^{+}, K^{-}\right)$allow to disentangle the contributions of the various quark flavors. 


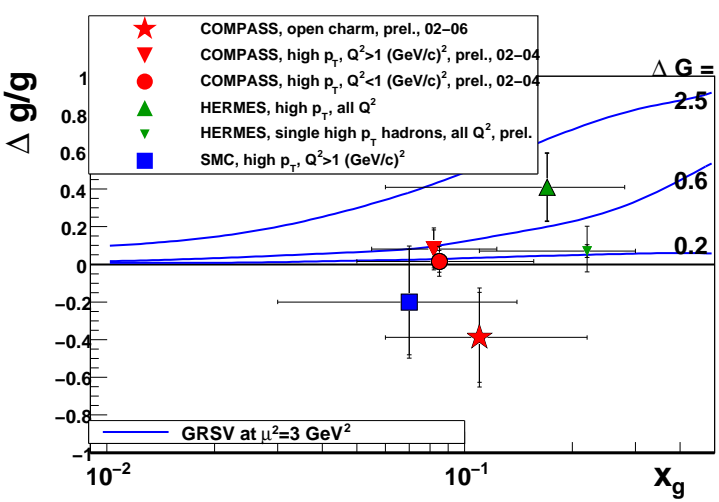

Fig. 5. Results from direct measurements of $\Delta g / g$.

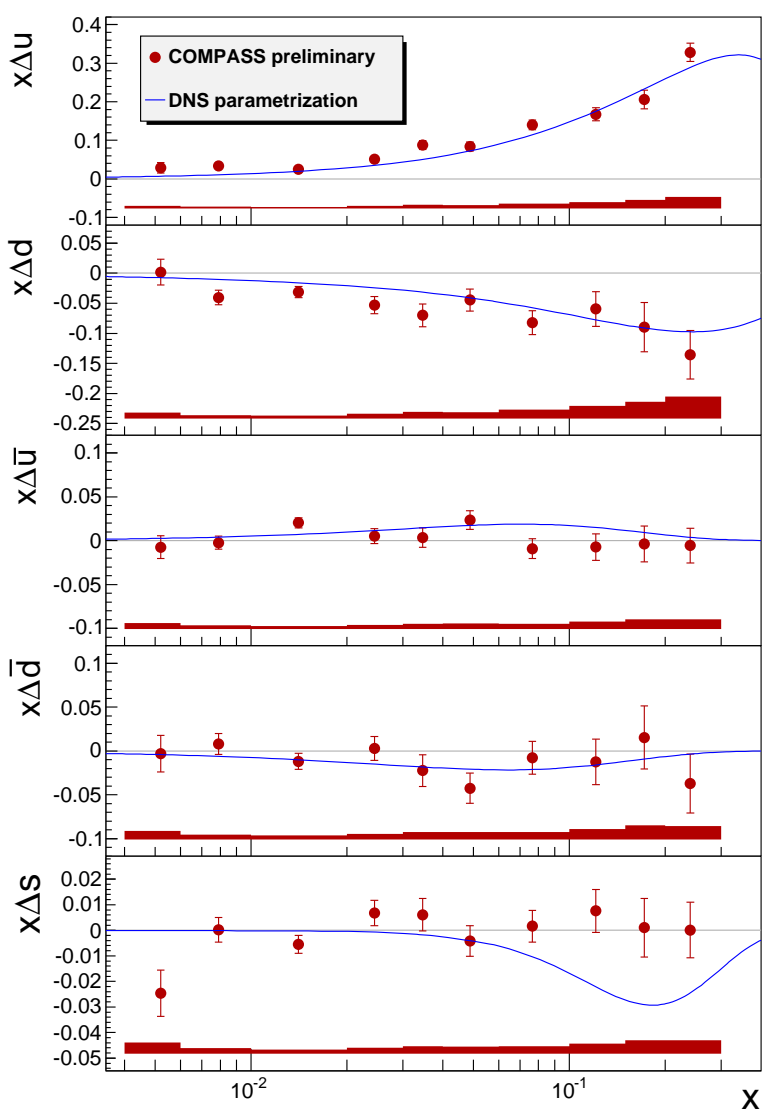

Fig. 6. Helicity distributions $\Delta q$ obtained from semi-inclusive asymmetries.

Figures. 7 and 8 show results obtained on a transversally polarized proton target. Figure 7 shows the Collins asymmetry which gives access to the transversity distribution. Figure 8 shows the Sivers asymmetry. Using this data together with corresponding asymmetries measured on the deuteron target allows an extraction of the transversity distribution, $\Delta_{T} q(x)$ and the Sivers function, $q_{T}[14,13]$.

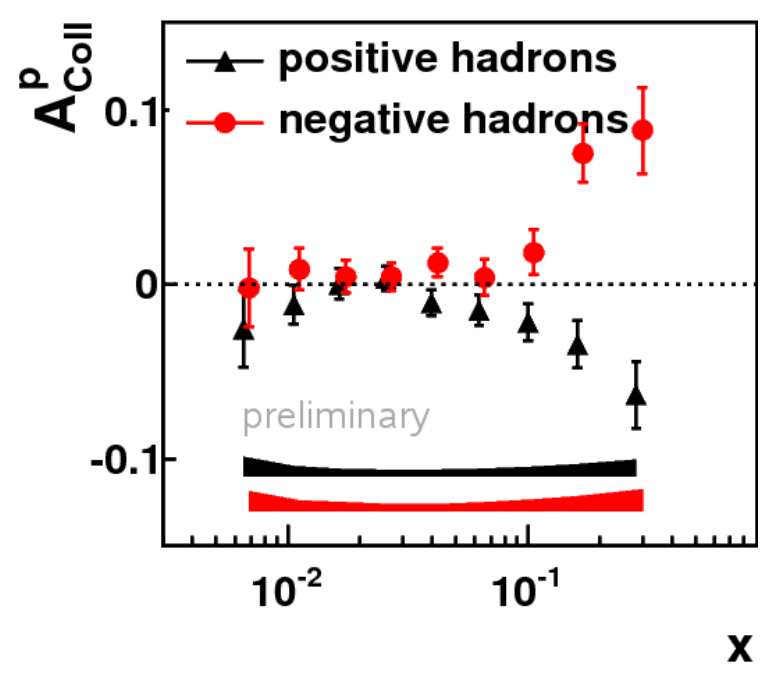

Fig. 7. Collins asymmetry from the proton target for positively and negatively charged hadrons.

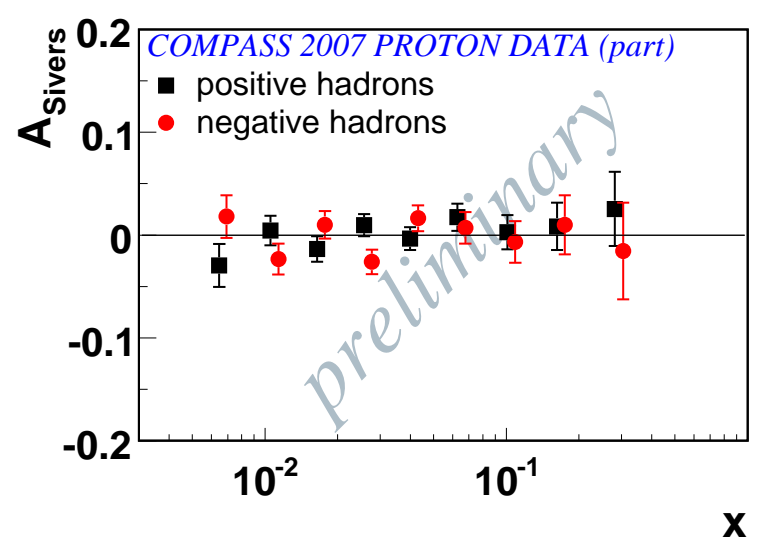

Fig. 8. Sivers asymmetry from the proton target for positively and negatively charged hadrons.

\section{Future plans at COMPASS}

We now turn to future plans of the COMPASS collaboration. The years 2010 and 2011 will be dedicated to run with longitudinal and transversely polarized proton target to improve the statistics of the measurements presented above. From 2012 on, we plan measurements of deep virtual Compton scattering and Drell-Yan processes.

\subsection{Deep Virtual Compton Scattering (DVCS)}

In deep virtual Compton scattering the following scattering process is studied

$$
\mu+p \rightarrow \mu^{\prime}+\gamma+p^{\prime}
$$

On the partonic level this process is shown in Fig. 9. The virtual photon is absorbed by a quark, the quark reemits a real photon and the proton stays intact. This picture indicates that one is on one hand looking at the partonic picture of the nucleon (absorbtions of a virtual photon of high 


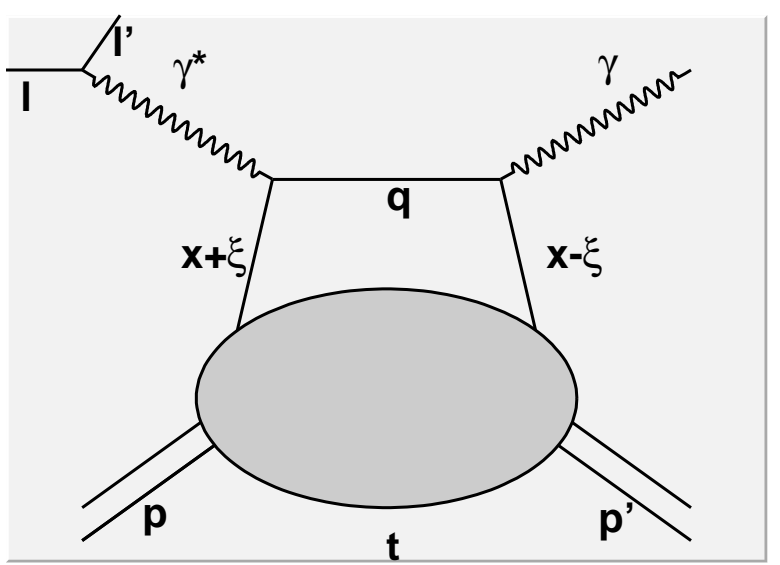

Fig. 9. The DVCS on the partonic level.

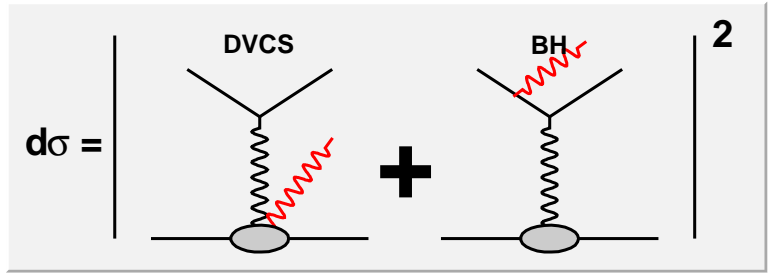

Fig. 10. Contribution to the process $\mu+p \rightarrow \mu^{\prime}+\gamma+p^{\prime}$

virtuality $\left.\left(Q^{2}>1 \mathrm{GeV}^{2}\right)\right)$ and the other hand a the spatial distribution as described by form factors measured in elastic scattering because the proton stays intact. Thus it is not surprising that DVCS is described by generalized parton distributions which are hybrids between parton distributions and from factors. These GPD depend on three variables (ignoring an additional dependence on a renormalization scale), the longitudinal momentum fraction $x$, the skewness $\xi$ and the momentum transfer squared to the nucleon $t$.

Unfortunately the DVCS process is not the only process contributing to the cross section; the Bethe Heitler $(\mathrm{BH})$ process has the same final state and thus interferes with the DVCS process as shown in Fig. 10.

The cross section can be expressed schematically as

$$
\begin{aligned}
\mathrm{d} \sigma= & \left(\mathrm{d} \sigma^{\mathrm{BH}}+\mathrm{d} \sigma_{\text {unpol }}^{\mathrm{DVCS}}+\mathrm{e}_{\mu} \mathrm{a}^{\mathrm{BH}} \mathcal{R e}\left(\mathrm{A}^{\mathrm{DVCS}}\right)\right) \times \cos (\mathrm{n} \Phi) \\
& +\left(P_{\mu} \mathrm{d} \sigma_{\text {pol }}^{\mathrm{DVCS}}+\mathrm{e}_{\mu} \mathrm{P}_{\mu} \mathrm{a}^{\mathrm{BH}} \mathcal{I} \mathrm{m}\left(\mathrm{A}^{\mathrm{DVCS}}\right)\right) \times \sin (\mathrm{n} \Phi)
\end{aligned}
$$

where $e_{\mu}\left(P_{\mu}\right)$ denotes the muon charge (polarization). $\Phi$ denotes the angle between the lepton and the $\gamma$-proton plane. The amplitudes appearing in eq. 2 are related to the GPDs via relations like

$$
A^{D V C S}=\int_{-1}^{1} \mathrm{dx} \frac{\mathrm{H}(\mathrm{x}, \xi, \mathrm{t})}{\mathrm{x}-\xi+\mathrm{i} \epsilon}
$$

Compass has at its disposal a negatively polarized positively charged muon beam or a positively polarized negatively charged muon beam. The cross section difference and sum of these two measurements project out various contributions in eq. 2 :

$$
\text { difference : } \mathrm{d} \sigma^{\mu^{+} \downarrow}-\mathrm{d} \sigma^{\mu^{-} \uparrow} \propto \mathcal{R e}\left(\mathrm{A}^{\mathrm{DVCS}}\right)
$$

sum $: \mathrm{d} \sigma^{\mu^{+} \downarrow}+\mathrm{d} \sigma^{\mu^{-} \uparrow} \propto \mathcal{I} \mathrm{m}\left(\mathrm{A}^{\mathrm{DVCS}}\right)$.

Figure 11 shows the statistical precision reachable in 140 days of data taking for the beam charge spin asymmetry defined by $A=\left(\mathrm{d} \sigma^{\mu^{+} \downarrow}-\mathrm{d} \sigma^{\mu^{-} \uparrow}\right) /\left(\mathrm{d} \sigma^{\mu^{+} \downarrow}+\mathrm{d} \sigma^{\mu^{-} \uparrow}\right)$. The curves are two models [10], the blue line assumes that $H$ factorizes in a quark distribution times a form factor and the red one assumes a strong correlation between the transverse position and longitudinal momentum fraction carried by the quark. Our measurement would clearly be able to distinguish the two models.

Concerning the kinematic range covered, COMPASS will be able to contribute in the the range form $x \approx 0.01$ and $x \approx 0.1$ ) not covered by any previous (H1, Zeus, HERMES) or future (JLAB12) experiment.

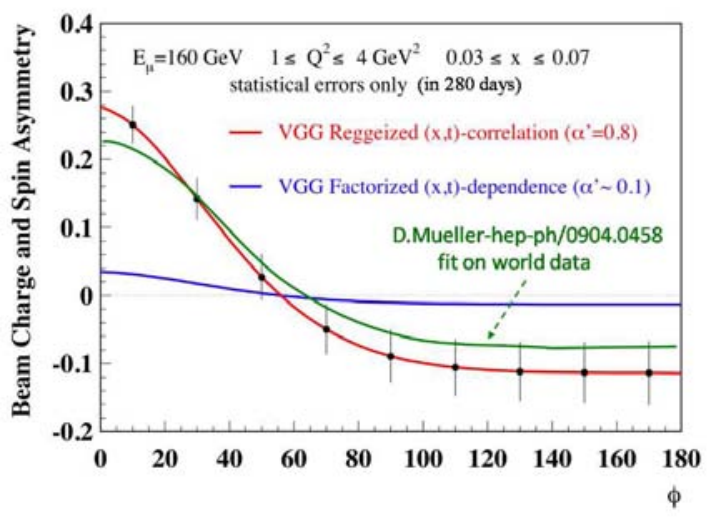

Fig. 11. Statistical precision reachable in 140 days for the beam charge spin asymmetry. The curves are two models [10-12] once assuming that $H$ factorizes in a quark distribution times a form factor and once assuming a strong correlation between the transverse position and longitudinal momentum fraction.

Figure 12 shows results from a first test measurement performed in 2008. The expected $\phi$ dependence (mostly originating from the Bethe-Heitler process) is clearly observed. The running conditions were: $160 \mathrm{GeV}$ muon beam on a $40 \mathrm{~cm}$ Liquid hydrogen target and one day of data taking.

In a first phase COMPASS plans to upgrade the spectrometer by installing a $2.5 \mathrm{~m}$ long liquid hydrogen target and a $4 \mathrm{~m}$ long recoil detector surrounding the target to detect the slow recoil proton in the DVCS reaction. In addition the calorimetry will be upgraded for a better detection of the photon. In a second phase, measurements with a polarized target giving access to the generalized parton distribution $\tilde{H}$ and $\tilde{E}$ are planed.

\subsection{Drell-Yan physics}

The Drell-Yan process

$$
\pi+p \rightarrow \mu^{+}+\mu^{-}+X
$$


EPJ Web of Conferences

Table 2. The Drell-Yan cross section in terms of various parton distribution functions.

\begin{tabular}{rlll}
\hline \multicolumn{2}{l}{ Unpolarized target: } & & \\
\hline $\mathrm{d} \sigma^{D Y} \propto \quad \ldots+\bar{h}_{1}^{\perp}\left(x_{1}, k_{T, 1}^{2}\right)$ & $\times$ & $h_{1}^{\perp}\left(x_{2}, k_{T, 2}^{2}\right) \cos (2 \Phi)$ \\
& Boer-Mulders & $\times$ & Boer-Mulders \\
\hline transversally polarized target: & & \\
\hline $\mathrm{d} \sigma^{D Y} \propto \quad$ & $\times$ & $f_{1 T}^{\perp}\left(x_{2}, k_{T, 2}\right) \sin \left(\Phi-\Phi_{S 2}\right)+$ \\
& $\bar{f}_{1}\left(x_{1}, k_{T, 1}^{2}\right)$ & $\times$ & Sivers \\
$\overline{\mathbf{q}}$ & $\times$ & $h_{1}\left(x_{2}, k_{T, 2}\right) \sin \left(\Phi+\Phi_{S 2}\right)+$ \\
$\bar{h}_{1}^{\perp}\left(x_{1}, k_{T, 1}^{2}\right)$ & $\times$ & Transversity \\
Boer-Mulders & $\times$ & $h_{1, T}^{\perp}\left(x_{2}, k_{T, 2}\right) \sin \left(3 \Phi-\Phi_{S 2}\right)$ \\
$\bar{h}_{1}^{\perp}\left(x_{1}, k_{T, 1}^{2}\right)$ & $\times$ & Pretzelosity \\
Boer-Mulders & $\times$
\end{tabular}

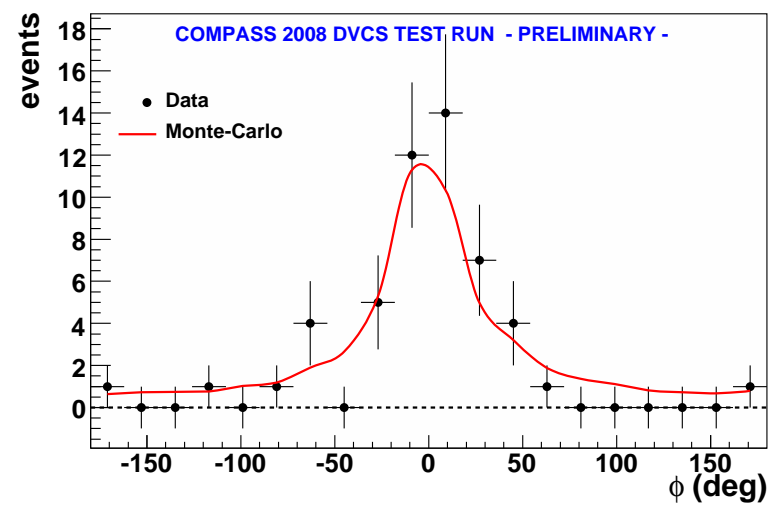

Fig. 12. Results from test run for the $\Phi$ distribution.

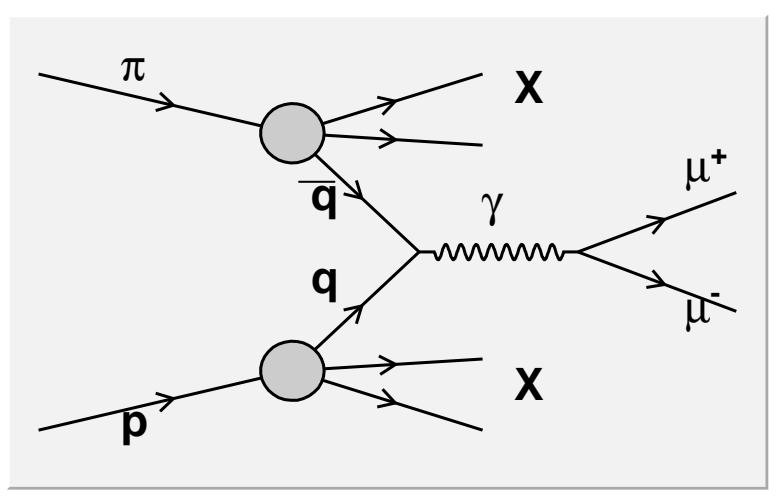

Fig. 13. The Drell-Yan process.

studied by COMPASS is depicted in Fig. 13. In leading twist the cross section can be written in terms of parton distribution functions as shown in Tab. 2. The angles $\Phi$ and $\Phi_{S 2}$ appearing in the cross section are defined in Figure 14. Note that in contrast to DIS, no fragmentation function appear in the cross section.

Of particular interest here is a relation between the Sivers and Boer-Mulders parton distributions measured in deep inelastic scattering and Drell-Yan. Both are naively

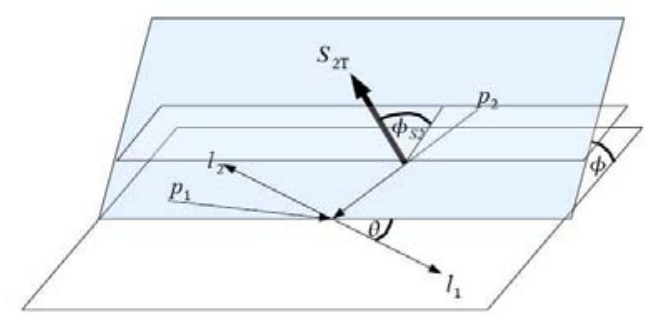

Fig. 14. Kinematics of the Drell-Yan process in the Collins-Soper frame. $S_{2 T}$ denotes the transverse polarization of the target proton.

time reversal odd and require an initial state interaction in the Drell-Yan process and a final state interaction in semiinclusive deep inelastic scattering (SIDIS) as first realized by Collins [17]. This leads to the following relations:

$$
\text { Sivers: } \quad f_{1 T}^{\perp}(D Y)=-f_{1 T}^{\perp}(S I D I S)
$$

Boer-Mulders: $\quad h_{1}^{\perp}(D Y)=-h_{1}^{\perp}(S I D I S)$

The verification of these relations would be an important test of our understanding of TMD distributions.

Exploiting the angular dependence of the cross section by measuring the following asymmetry gives for example access to the Sivers function

$$
A_{U T}=\frac{\int_{0}^{2 \pi}\left(\mathrm{d} \sigma^{\uparrow}-\mathrm{d} \sigma^{\downarrow}\right) \sin \left(\Phi_{S 2}-\Phi\right) \mathrm{d} \Phi}{\int_{0}^{2 \pi}\left(\mathrm{d} \sigma^{\uparrow}+\mathrm{d} \sigma^{\downarrow}\right) \mathrm{d} \Phi}
$$

Figure 15 shows the statistical precision reachable on $A_{U T}$ assuming 2 years of data taking.

For the Drell-Yan measurement it is mandatory to detect muon pairs in the final state. Fig. 16 shows reconstructed muon pairs from a 2007 test run. The number of measured $J / \Psi$ s agrees with the expected number of $20 \pm 8$. The experimental upgrades needed for this measurement are a hadron absorber downstream of the target and a new trigger system to trigger on $\mu^{+}-\mu^{-}$pairs. 


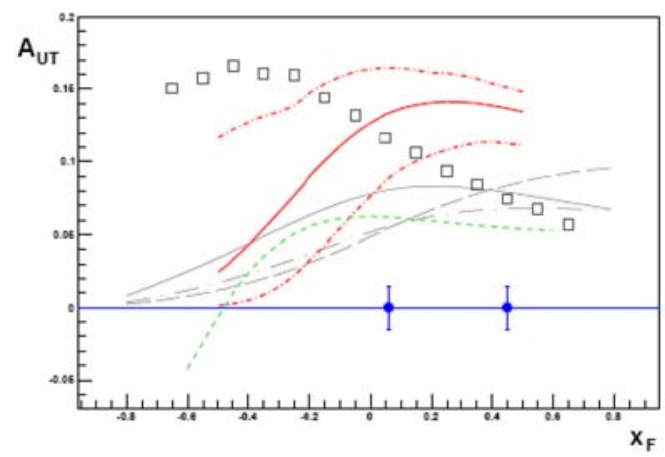

Fig. 15. Statistical precision on $A_{U T}$ reachable in 2 years of data taking. The curves and boxes are parameterizations by $[17,16,19$, $18,20]$.

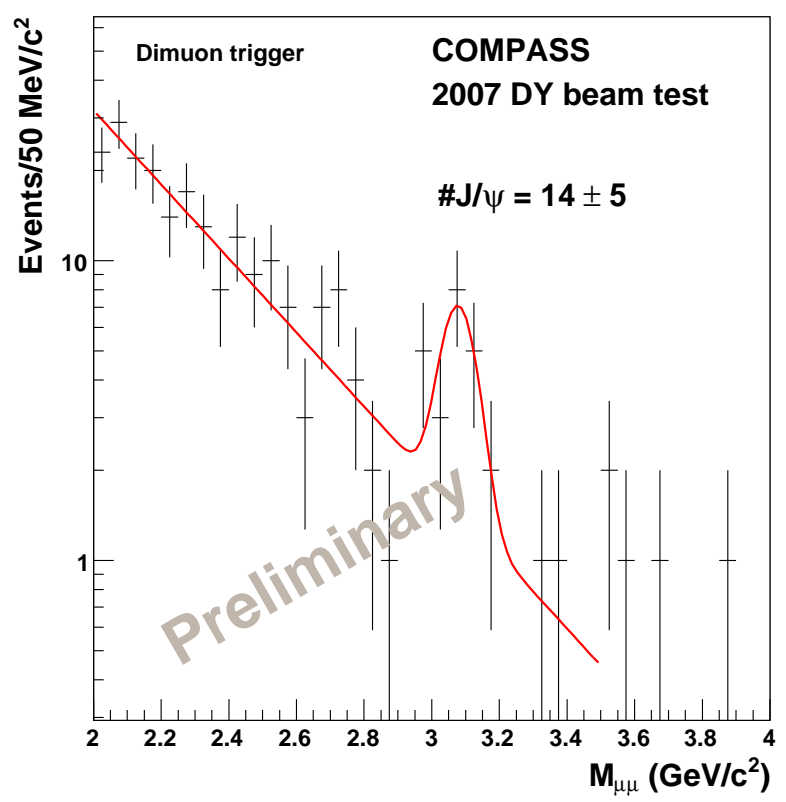

Fig. 16. Reconstructed muon pairs from a 2007 test run. The number of measured $J / \Psi_{\mathrm{s}}$ agrees with the expected number of $20 \pm 8$.

\section{References}

1. R. Hofstadter and R. W. McAllister, Phys. Rev. 98 (1955) 217.

2. M. Breidenbach et al., Phys. Rev. Lett. 23 (1969) 935.

3. X. Ji, Ann. Rev. Nucl. Part. Sci. 54 (2004) 413.

4. P. Abbon et al. [COMPASS Collaboration], Nucl. Instrum. Meth. A 577 (2007) 455 [arXiv:hepex/0703049].

5. M. Alekseev et al. [COMPASS Collaboration], arXiv:0904.3209 [hep-ex]. Phys. Lett. B 676 (2009) 31

6. J. Collins PLB536, 43 (2002)

7. D. Boer and P. J. Mulders, Phys. Rev. D 57 (1998) 5780 [arXiv:hep-ph/9711485].
8. A. Bacchetta, M. Diehl, K. Goeke, A. Metz, P. J. Mulders and M. Schlegel, JHEP 0702 (2007) 093 [arXiv:hep-ph/0611265].

9. M. Alekseev et al. [COMPASS Collaboration], Phys. Lett. B 680 (2009) 217 [arXiv:0905.2828 [hep-ex]].

10. M. Vanderhaeghen, P. A. M. Guichon and M. Guidal, Phys. Rev. Lett. 80 (1998) 5064.

11. M. Vanderhaeghen, P. A. M. Guichon and M. Guidal, Phys. Rev. D 60 (1999) 094017 [arXiv:hep$\mathrm{ph} / 9905372]$.

12. K. Goeke, M. V. Polyakov and M. Vanderhaeghen, Prog. Part. Nucl. Phys. 47 (2001) 401 [arXiv:hep$\mathrm{ph} / 0106012]$.

13. M. Anselmino et al., arXiv:0807.0166 [hep-ph].

14. M. Anselmino, M. Boglione, U. D’Alesio, A. Kotzinian, F. Murgia, A. Prokudin and S. Melis, Nucl. Phys. Proc. Suppl. 191 (2009) 98 [arXiv:0812.4366 [hep-ph]].

15. A. Bacchetta, AIP Conf. Proc. 1149 (2009) 447 [arXiv:0902.2712 [hep-ph]].

16. A. V. Efremov, K. Goeke, S. Menzel, A. Metz and P. Schweitzer, Phys. Lett. B 612 (2005) 233 [arXiv:hep-ph/0412353].

17. J. C. Collins, A. V. Efremov, K. Goeke, S. Menzel, A. Metz and P. Schweitzer, Phys. Rev. D 73 (2006) 014021 [arXiv:hep-ph/0509076].

18. M. Anselmino, M. Boglione, U. D’Alesio, S. Melis, F. Murgia and A. Prokudin, Phys. Rev. D 79 (2009) 054010 [arXiv:0901.3078 [hep-ph]].

19. A. Bianconi and M. Radici, Phys. Rev. D 73 (2006) 114002 [arXiv:hep-ph/0602103].

20. A. Bacchetta, F. Conti and M. Radici, Phys. Rev. D 78 (2008) 074010 [arXiv:0807.0323 [hep-ph]]. 